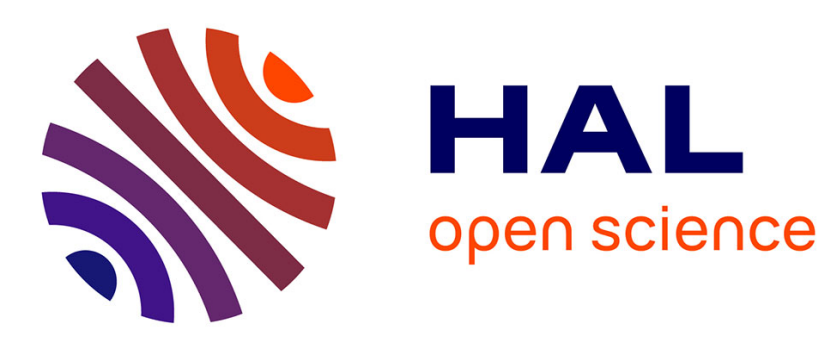

\title{
Simultaneous Containment of Several Polygons: Analysis of the Contact Configurations
}

\author{
Olivier Devillers
}

\section{To cite this version:}

Olivier Devillers. Simultaneous Containment of Several Polygons: Analysis of the Contact Configurations. International Journal of Computational Geometry and Applications, 1993, 3 (4), pp.429-442. 10.1142/S0218195993000270 . inria-00167170

\section{HAL Id: inria-00167170 https://hal.inria.fr/inria-00167170}

Submitted on 16 Aug 2007

HAL is a multi-disciplinary open access archive for the deposit and dissemination of scientific research documents, whether they are published or not. The documents may come from teaching and research institutions in France or abroad, or from public or private research centers.
L'archive ouverte pluridisciplinaire HAL, est destinée au dépôt et à la diffusion de documents scientifiques de niveau recherche, publiés ou non, émanant des établissements d'enseignement et de recherche français ou étrangers, des laboratoires publics ou privés. 
published in International Journal of Computational Geometry \& Applications, 3:429-442, 1993

\title{
Simultaneous Containment of Several Polygons: Analysis of the Contact Configurations
}

\author{
Olivier Devillers
}

\begin{abstract}
The main concern of this paper is the detection of double contact configurations for some polygons moving in translation in a polygonal environment. We first establish some general properties about such configurations and give conditions of existence of double contacts for two or three objects.

For three convex polygons moving in a polygonal environment or three simple polygons moving in a rectangle there always exists a double contact. Two examples without possibility of double contacts are given, one with three polygons (not convex) moving in a polygonal environment, and one with four convex polygons moving in a rectangle.

We deduce an algorithm detecting a double contact position in time $O\left(n^{2}\right)$ (resp. $\left.O\left(n^{3}\right)\right)$ for two (resp. three) convex polygons of constant sizes moving in a non-convex polygon of size $n$.

keywords: Motion planning, polygon containment, robotics.
\end{abstract}

\section{Introduction}

This paper addresses the problem of detecting collision-free positions of polygonal shapes translating in the plane among polygonal obstacles. More precisely, we are only looking for a special kind of positions (called double contacts) and we study the conditions of existence of theses special positions.

In the following, the environment refers to a polygonal region of the plane which is the set of obstacles, and we call a configuration of a given polygonal object a translation moving this object from its reference position to another position in the plane. A configuration is said free if the translated polygon does not intersect the environment. The set of free configurations is called the free space of the object. At this point, two kind of problems can be studied:

\footnotetext{
${ }^{0}$ INRIA, BP 93, 06902 Sophia Antipolis cedex, France. E-mail: Firstname.Lastname@sophia.inria.fr . This work was partially supported by ESPRIT Basic Research Action Nr. 3075 (ALCOM).
} 
the determination of the whole free space, or just the computation of one free configuration if it exists. We focus on the second point in this paper.

We are interested in the placement of several polygons. A configuration for such a system is a translation for each polygon, and this configuration is free if the translated polygons do not intersect the environment and if they do not intersect each others.

Several results have been obtained concerning this problem. The free space of two convex polygons moving in a convex environment can be found in linear time.[1] In the case of two or three general polygons in general polygonal environment Avnaim and Boissonnat [2,3] gave algorithms to compute the free space or to only find one solution. The complexity depends on the different cases of convexity of objects and environment, in particular, they found one solution for the containment of two (resp. three) convex polygons in a general polygon in $O\left(n^{2} \log ^{2} n\right)\left(\right.$ resp. $\left.O\left(n^{3} \log ^{2} n\right)\right)$ if $n$ is the environment size and the sizes of the objects are assumed to be constant. ${ }^{1}$ Some work had also be done on special case such as rectilinear polygons.[4]

In a system of $q$ polygons, a configuration of the system is described by $q$ translations in the plane, that is by a vector of $\mathbb{R}^{2 q}$, and the free space is a polygonal domain of $\mathbb{R}^{2 q}$. If some vertex of an object touches an edge of another object (or of the environment), we call this configuration a contact configuration and the pair vertex-edge is the label of the contact. Such a contact configuration necessarily lies on the boundary of the free space since a small motion can move the vertex on the forbidden side of the edge; in fact, if there is only one contact (one vertex-edge pair) the configuration lies on a facet of the free space, and if more contacts are involved, the configuration lies on a face of lower dimension. In general position, a vertex of the free space corresponds to $2 q$ contacts.

A contact configuration having two labels involving the same pair of objects (or the same object and the environment) is called a double contact. This paper presents some results about contact configurations, and particularly about double contacts. If $q=1$ there is only one possibility of contact (objectenvironment) and the vertices of the free space correspond to two contacts, thus they are necessarily double contacts. If $q=2$ there are three possibilities of contacts (object1-environment, object2-environment, object1-object2) since vertices of the free space involves four contacts, one of the three pairings of objects must appear twice, so vertices are double contacts. The interesting case arises when $q \geq 3$. This paper studies the possibility for the free space to be non-empty without existence of double contacts.

This paper proves that for three convex polygons in a polygonal environment

\footnotetext{
${ }^{1}$ In

fact, if $m_{i}$ is the size of polygon number $i$, the complexity is $O\left(n^{2} m_{1} m_{2} \log n m_{1} \log n m_{2}\right)$ for two objects and

$O\left(n^{3} m_{1} m_{2} m_{3}\left(\left(m_{1}+m_{2}\right)\left(m_{1}+m_{3}\right) \log m_{1} m_{2} m_{3}+\log ^{2} n m_{1} m_{2} m_{3}\right)\right)$ for three objects. If we assume that $m_{i}=O(m)$ and $m<n$, this can be simplified in $O\left(n^{2} m^{2} \log ^{2} n\right)$ and $O\left(n^{3} m^{3} \log ^{2} n+n^{3} m^{5} \log m\right)$.
} 
or for three polygons moving in a rectangle there always exists double contacts (if the free space is not empty). Algorithms are deduced for two or three convex polygons moving in a polygonal environment; if $n$ is the environment size and the sizes of the objects are assumed to be constant the complexity of determining a double contact configuration is $O\left(n^{2}\right)$ (resp. $O\left(n^{3}\right)$ ) in the case of two (resp. three) objects, these complexities improve those of Avnaim and Boissonnat by a $\log ^{2} n$ factor. ${ }^{2}$

In general this property is false, and examples of systems where there does not exist double contacts are given for three non-convex polygons in a nonconvex environment and for four convex polygons in a rectangle.

The paper is organized as follows: Section 2 introduces some notations, Section 3 describes the boundary of the free space, Section 4 studies the double contact configurations, and finally some algorithmic results are deduced in Section 5 .

\section{Notations and Classical Results}

We consider polygonal objects moving in translation in a polygonal environment. The closure of the environment is denoted by $\mathcal{O}$ and the closure of the objects by $\mathcal{S}_{i}(i=1 \ldots q)$. The size of $\mathcal{O}\left(\right.$ resp. $\left.\mathcal{S}_{i}\right)$ is denoted by $n$ (resp. $\left.m_{i}\right)$. The position of each object is characterized by the translation $c_{i} \in \mathbb{R}^{2}$ which moves $\mathcal{S}_{i}$ from its reference position to its current position. The translation $c_{i}$ is called a configuration of $\mathcal{S}_{i}$. The object $\mathcal{S}_{i}$ in the configuration $c_{i}$ is denoted by $\mathcal{S}_{i}^{c_{i}}$. The usual way to represent a position for a system of $q$ objects is to use a $2 q$-dimensional space: the configuration space. A point of this space, $c=\left(c_{1}, \ldots, c_{q}\right) \in \mathbb{R}^{2 q}$, is a configuration of the complete system.

Now we define a free configuration as a configuration where the closures of the objects have no intersections. We also define a contact configuration as a configuration where the boundaries of the objects intersect but their interiors do not intersect. We use the following definitions of Avnaim[3]:

$$
\begin{aligned}
\mathcal{L}= & \left\{c=\left(c_{1}, \ldots, c_{q}\right), \forall i \mathcal{S}_{i}^{c_{i}} \cap \mathcal{O}=\emptyset \text { and } \forall i, j i \neq j, \mathcal{S}_{i}^{c_{i}} \cap \mathcal{S}_{j}^{c_{j}}=\emptyset\right\} \\
& \text { is the free space } \\
\mathcal{C}= & \left\{c=\left(c_{1}, \ldots, c_{q}\right), c \notin \mathcal{L}, \forall i \breve{\mathcal{S}_{i}^{c_{i}}} \cap \breve{\mathcal{O}}=\emptyset \text { and } \forall i, j i \neq j, \breve{\mathcal{S}_{i}^{c_{i}}} \cap \breve{\mathcal{S}_{j}^{c_{j}}}=\emptyset\right\}
\end{aligned}
$$

is the contact space $(\breve{\mathcal{A}}$ denote the interior of a set $\mathcal{A}$ )

$(\mathcal{C}$ is also the boundary of $\mathcal{L})$

\footnotetext{
${ }^{2}$ If

the size of the polygons is not constant, the time bounds are $O\left(n^{2} m_{1} m_{2} \log n m_{1} \log n m_{2}\right)$ for two objects and $O\left(\sum_{i \neq j \neq k \neq i} n m_{i}\left(n+m_{i}\right)^{2} m_{j} m_{k} \log m_{j} m_{k}\right)$ for three objects. If we assume $m_{i}=O(m)$ and $m=O(n)$, we get $O\left(n^{2} m^{2} \log ^{2} n\right)$ and $O\left(n^{3} m^{3} \log m\right)$.
} 

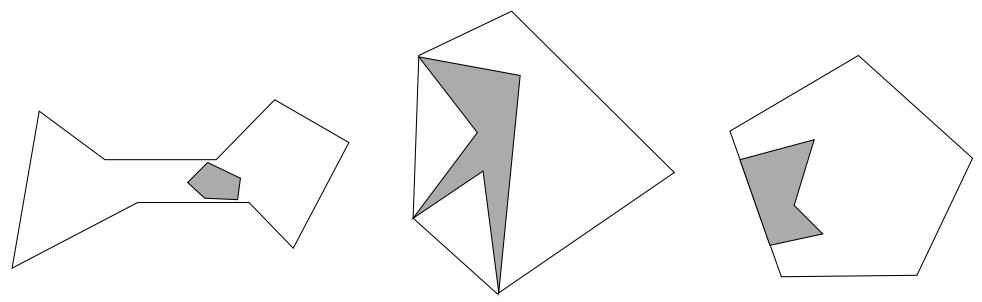

Figure 1: Some degenerate cases

$$
\begin{aligned}
\mathcal{L}_{i}= & \left\{c_{i}, \mathcal{S}_{i}^{c_{i}} \cap \mathcal{O}=\emptyset\right\} \\
& \text { is the free space of only one object } \mathcal{S}_{i} \text { in the environment } \mathcal{O} \\
\mathcal{L}_{i j}= & \left\{c_{i}-c_{j}, \mathcal{S}_{i}^{c_{i}} \cap \mathcal{S}_{j}^{c_{j}}=\emptyset\right\} \\
& \text { is the free space of } \mathcal{S}_{i} \text { relatively to } \mathcal{S}_{j} \\
\mathcal{U}_{i j}= & \left\{c_{i}-c_{j}, c_{i} \in \mathcal{L}_{i}, c_{j} \in \mathcal{L}_{j}, c_{i}-c_{j} \in \mathcal{L}_{i j}\right\} \\
& \text { is the set of relative free configurations of } \mathcal{S}_{i} \text { and } \mathcal{S}_{j} \text { in } \mathcal{O} \\
\mathcal{R}_{i j}= & \left\{c_{i}-c_{j}, \exists c \in \mathcal{L}, c_{i} \text { and } c_{j} \text { are the } i^{\text {th }} \text { and } j^{\text {th }} \text { components of } c\right\} \\
& \text { is the set of relative free configurations of } \mathcal{S}_{i} \text { and } \mathcal{S}_{j} \text { in the complete } \\
& \text { system of } q \text { objects in the environment } \mathcal{O}
\end{aligned}
$$

We assume without loss of generality that the system is in general position; a precise definition will be given in Section 3.1. Degenerate cases such as constrained motion in a corridor or parallelism between objects are therefore ruled out (see Figure 1). A small deformation of the objects and the environment can be used to put a degenerate system in general position.

For a polygonal system, all the sets defined above are polyhedra; these polyhedra are not necessarily convex. $\mathcal{L}_{i}, \mathcal{L}_{i j}, \mathcal{U}_{i j}$ and $\mathcal{R}_{i j}$ are open polygons. $\mathcal{L}$ is an open polyhedron of dimension $2 q$, and $\mathcal{C}$ is its boundary.

An usual way to compute $\mathcal{L}_{i}$ and $\mathcal{L}_{i j}$ is to use the Minkowski difference, denoted by $\ominus$ (see, for example, the article by Lozano-Perez and Wesley[5]). The Minkowski difference of two sets $\mathcal{F}$ and $\mathcal{G}$ is $\mathcal{F} \ominus \mathcal{G}=\{f-g, f \in \mathcal{F}, g \in \mathcal{G}\}$, so $c \in \mathcal{F} \ominus \mathcal{G}$ means that the translated polygon $\mathcal{G}^{c}$ intersects $\mathcal{F}$ (if $c=f-g$, then $\left.f=c+g \in \mathcal{F} \cap \mathcal{G}^{c}\right)$. Thus, we can deduce $(\bar{A}$ denoting the complementary set of $A$ ):

$$
\begin{aligned}
\overline{\mathcal{L}}_{i} & =\mathcal{O} \ominus \mathcal{S}_{i}, \\
\overline{\mathcal{L}_{i j}} & =\mathcal{S}_{j} \ominus \mathcal{S}_{i} .
\end{aligned}
$$

The Minkowski difference of two polygons of sizes $n$ and $m$ can be computed in time $O\left(n^{2} m^{2}\right)$ and has a size $O\left(n^{2} m^{2}\right)$. This bound is tight in the worst case. [2] 
This computation can be done as follows. $\mathcal{F} \ominus \mathcal{G}$ is a polygon whose edges are supported either by edges of $\mathcal{F} \ominus g$ or $f \ominus \mathcal{G}$ for $f$ vertex of $\mathcal{F}$ and $g$ vertex of $\mathcal{G}$. It is possible to compute the arrangement of these $O(m n)$ line segments in $O\left(m^{2} n^{2}\right)$ time[6] and then select the edges of $\mathcal{F} \ominus \mathcal{G}$ among the edges of this arrangement in a straightforward manner.

If $\mathcal{F}$ is a general polygon and $\mathcal{G}$ is a convex polygon, the result can be computed in time $O(n m \log n m)$ and has a size $O(n m)$.[7] This algorithm computes the Voronoi diagram of the environment for the convex metric associated with the convex polygon, thus the free space is exactly the set of points at unit distance from the environment for this metric. In the case of two convex polygons, we can achieve the computation in time $O(n+m)$ and the size is also $O(n+m) \cdot[1]$

Avnaim[2] has established the following results:

$$
\begin{aligned}
\mathcal{U}_{i j}= & \mathcal{L}_{i j} \cap\left(\mathcal{L}_{i} \ominus \mathcal{L}_{j}\right), \\
\mathcal{R}_{i j} \subset \mathcal{U}_{i j} \cap\left(\mathcal{U}_{i k} \ominus \mathcal{U}_{j k}\right), & \\
& \text { where } i \neq j \neq k \neq i,
\end{aligned}
$$

and in the special case of three objects in a parallelogram:

$$
\begin{aligned}
\mathcal{R}_{i j}= & \mathcal{U}_{i j} \cap\left(\mathcal{U}_{i k} \ominus \mathcal{U}_{j k}\right), \\
& \text { where } i, j, k \in\{1,2,3\}, i \neq j \neq k \neq i .
\end{aligned}
$$

\section{The Contact Space}

As stated in Section 2.1, the configuration space has dimension $2 q$ and the free-space is a polyhedron in this space.

\subsection{Contact Configuration}

A configuration of $\mathcal{C}$ is called a contact (see Section 2.1). Corresponding to a contact $c=\left(c_{1}, \ldots, c_{q}\right)$, there exist two objects $\mathcal{S}_{i}$ and $\mathcal{S}_{j}$ (or an object $\mathcal{S}_{i}$ and the environment $\mathcal{O}$ ) such that $\mathcal{S}_{i}^{c_{i}}$ and $\mathcal{S}_{j}^{c_{j}}\left(\mathcal{S}_{i}^{c_{i}}\right.$ and $\mathcal{O}$ ) have a common point on their boundary. We assume without loss of generality that this point corresponds to a vertex $V$ of $\mathcal{S}_{i}$ and to an edge $e$ of $\mathcal{S}_{j}$. Such an instance of a contact $(V, e)$ is called label of simple contact.

Now let $(V, e)$ be a label of simple contact, it constrains the relative motion between $\mathcal{S}_{i}$ and $\mathcal{S}_{j}$ such that the vertex $V$ of $\mathcal{S}_{i}$ slides on the edge $e$ of $\mathcal{S}_{j}$. This imposes a linear constraint on the two dimensional vector $c_{i}-c_{j}$, for the points in the contact space $\mathcal{C}$. Therefore, the contact space $\mathcal{C}$ is supported by an hyperplane corresponding to the linear constraint on $c_{i}-c_{j}$ due to the label of simple contact $(V, e)$. Such a supporting hyperplane is called a contact hyperplane.

We can now define what a system in general position means. 
Definition 1 The system, composed of an environment $\mathcal{O}$ and $q$ polygons moving in translation, is said to be in general position if, in the arrangement of all contact hyperplanes in the $2 q$-dimensional space, the intersection of $k \leq 2 q$ hyperplanes is a subspace of dimension $2 q-k$ and the intersection of $k>2 q$ hyperplanes is empty.

This definition means that redundancies like confounded hyperplanes or three hyperplanes meeting at a same ridge are forbidden, but parallelism between hyperplanes are not.

As claimed in Section 2.1, a small perturbation of objects can turn any system in general position. [8] In this case $\mathcal{C}$ is the boundary of $\mathcal{L}$.

Now we introduce a few definitions. A simple contact $c \in \mathcal{C}$ is a point of $\mathcal{C}$ lying on a contact hyperplane. A $k$-contact is a contact $c \in \mathcal{C}$ lying on $k$ contact hyperplanes. A $k$-double contact is a $k$-contact $c \in \mathcal{C}$ such that two hyperplanes containing $c$ have labels involving the same pair of objects, otherwise it is a $k$-simple contact.

Let $c$ be a point on an hyperplane corresponding to a label of simple contact $(V, e)$. Let $E$ and $E^{\prime}$ be the endpoints of $e$. The range of $c \operatorname{such}$ that $V$ is between $E$ and $E^{\prime}$ is called the validity slab of the hyperplane. Any point of $\mathcal{C}$ on this validity slab is a contact configuration with label $(V, e)$. The points on the boundary of the validity slab are vertex-vertex contacts $(V, E)$ and $\left(V, E^{\prime}\right)$. A vertex-vertex contact $(V, E)$ corresponds to two labels of simple contact: $(V, e)$ and $\left(V, e^{\prime}\right)$,where $E$ is the common endpoint of edges $e$ and $e^{\prime}$. Such a contact is called a 2 -double contact. ${ }^{3}$

Usual properties of arrangements and the general position hypothesis yield the following proposition:

Proposition $2 \mathcal{C}$ is a polyhedron of dimension $2 q$, whose $(2 q-k)$-faces of $\mathcal{C}$ correspond to $k$-contacts.

\section{$3.2 \quad$ Vertices of $\mathcal{C}$}

This section establishes conditions under which a vertex of $\mathcal{C}($ or $\mathcal{L})$ is concave or convex. ${ }^{4} \mathrm{~A}$ first examination of the geometry of $\mathcal{C}$ allows us to conclude that a concave vertex of $\mathcal{C}$ is necessarily a vertex-vertex contact, so it can be reduced to a 2-dimensional problem since only the relative motion of two objects is relevant.

Consider a vertex $c \in \mathcal{C}$. Note that $c$ is a 0 -face and therefore, by Proposition 2 , it is a $2 q$-contact. So, $c$ is the intersection of $2 q$ contact hyperplanes. The vertex $c$ could be either concave or convex depending on the geometry of $\mathcal{L}$

\footnotetext{
${ }^{3}$ These two double contacts $(V, E)$ and $\left(V, E^{\prime}\right)$ define two subspace of $\mathbb{R}^{2 q}$ of dimension $2 q-2$ and having the same direction. They are two parallel ridges included in the hyperplane corresponding to the contact $(V, e)$.

${ }^{4}$ We define a concave vertex as a non-convex vertex. A vertex is convex if in a neighbourhood the polyhedron is an intersection of half-spaces.
} 


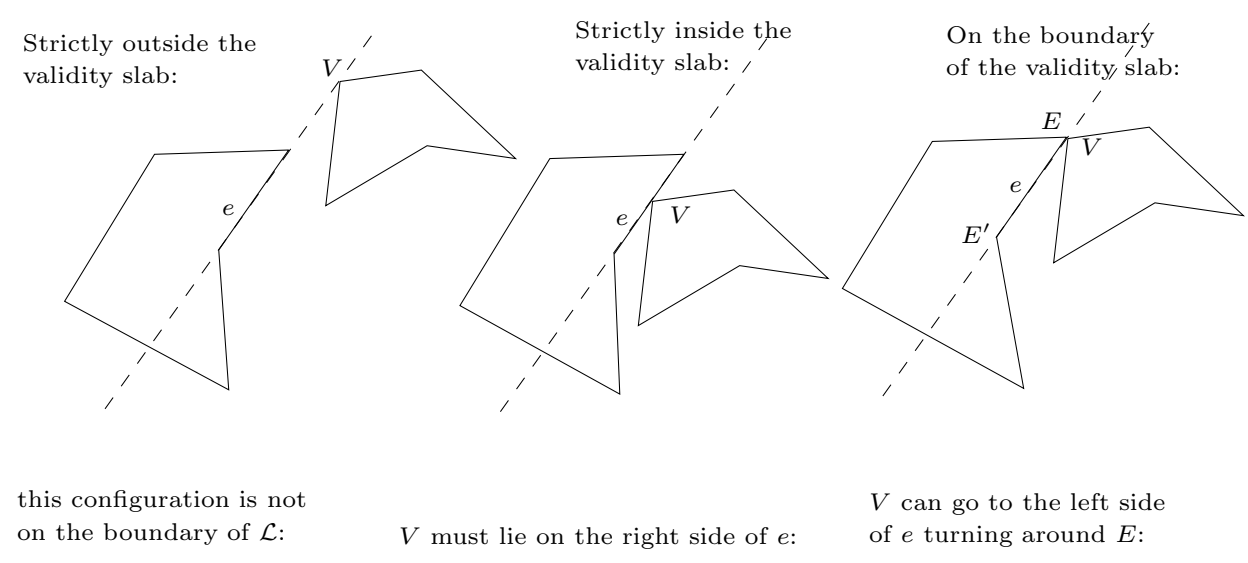

Figure 2: Configuration in the contact hyperplane.

in the neighbourhood of $c$. Consider whithout loss of generality one of the $2 q$ labels of simple contact $(V, e)$ defining $c$, with $V$ a vertex of $\mathcal{S}_{i}$ and $e$ an edge of $\mathcal{S}_{j} ; H$ denotes the contact hyperplane corresponding to $(V, e)$. Since $(V, e)$ is a contact, $c$ lies in $H$; moreover $c$ must be in the validity slab of $H$ (see Figure 2 ). If $c$ is not on the boundary of the validity slab, then, in a neighbourhood of $c, \mathcal{L}$ is included in a half-space limited by $H$, otherwise $c$ is on the boundary of the validity slab and thus corresponds to a vertex-vertex contact. If $c$ is not a vertex-vertex contact, then for each of the $2 q$ hyperplanes defining $c, \mathcal{L}$ is locally included in the corresponding half-spaces and thus in a neighbourhood of $c, \mathcal{L}$ is an intersection of half-spaces, and $c$ is convex.

Proposition 3 If a vertex $c$ of $\mathcal{C}$ is concave then it corresponds to a double contact between two vertices of two objects (or one vertex of an object and one vertex of the environment). 


\section{Double Contacts}

$\mathcal{C}$ is a polyhedron whose vertices correspond to $2 q$-contact configurations. Each of these vertices may be a $2 q$-simple contact or a double contact. We are interested in detecting the existence of double contacts. In other words, we want to solve the following question: is there a connected subset of $\mathcal{C}$ whose vertices are all $2 q$-simple contacts?

\subsection{Two Polygons}

In the case of only two objects, a vertex of $\mathcal{C}$ is a 4-contact, and a vertex of $\mathcal{C}$ is a 4-contact. Since there are only three different possibilities of contacts $\left(\mathcal{O}-\mathcal{S}_{1}, \mathcal{O}-\mathcal{S}_{2}, \mathcal{S}_{1}-\mathcal{S}_{2}\right)$, a 4 -contact is necessarily a double contact.

\subsection{Three Polygons}

In this section, we show that a system of three polygons in a polygonal environment need not have a double contact. Consider a system of three simple polygonal objects in a polygonal environment. A vertex of $\mathcal{C}$ is a 6-contact. There are exactly six different possibilities of contacts: $\mathcal{O}-\mathcal{S}_{1}, \mathcal{O}-\mathcal{S}_{2}, \mathcal{O}-\mathcal{S}_{3}$, $\mathcal{S}_{1}-\mathcal{S}_{2}, \mathcal{S}_{1}-\mathcal{S}_{3}, \mathcal{S}_{2}-\mathcal{S}_{3}$. We show that there does not exist a double contact in such a system in the following discussion.

We assume that a connected component of $\mathcal{C}$ does not contain double contact. A vertex $c$ of this component is a 6 -simple contact.

Let $\left(l_{\mathcal{O S}}, l_{\mathcal{O S}}, l_{\mathcal{O S}}, l_{\mathcal{S}_{1} \mathcal{S}_{2}}, l_{\mathcal{S}_{1} \mathcal{S}_{3}}, l_{\mathcal{S}_{2} \mathcal{S}_{3}}\right)$ be the label describing this contact $\left(l_{\mathcal{F G}}\right.$ is a label of simple contact between $\mathcal{F}$ and $\left.\mathcal{G}\right) . \quad c$ is the intersection of the six contact hyperplanes corresponding to these labels. From Proposition 2 and the discussion of Section 3.2, the $(6-k)$-faces incident to $c$ are defined as intersection of $k$ of these 6 contact hyperplanes. In particular, moving the configuration along an edge incident to $c$ corresponds to moving all the objects such that 5 of the simple contacts remain valid, and only one contact is broken. We choose the edge $e$ having $\left(l_{\mathcal{O S}}, l_{\mathcal{O S}}, l_{\mathcal{O S}}, l_{\mathcal{S}_{1} \mathcal{S}_{3}}, l_{\mathcal{S}_{2} \mathcal{S}_{3}}\right)$ as label of 5 -simple contact. When the configuration moves along this edge, the contact between $\mathcal{S}_{1}$ and $\mathcal{S}_{2}$ disappears.

Let $c^{\prime}$ be the other endpoint of $e$. To agree with our hypothesis, $c^{\prime}$ is a 6simple contact different from $c$, its label is $\left(l_{\mathcal{O S}}, l_{\mathcal{O S}}, l_{\mathcal{O S}}, l_{\mathcal{S}_{1} \mathcal{S}_{2}}^{\prime}, l_{\mathcal{S}_{1} \mathcal{S}_{3}}, l_{\mathcal{S}_{2} \mathcal{S}_{3}}\right)$ with $l_{\mathcal{S}_{1} \mathcal{S}_{2}} \neq l_{\mathcal{S}_{1} \mathcal{S}_{2}}^{\prime}$. ¿From $c^{\prime}$ it is possible to get the edge $e^{\prime}$ having label $\left(l_{\mathcal{O S}}\right.$, $\left.l_{\mathcal{O S}}, l_{\mathcal{O S}}, l_{\mathcal{S}_{1} \mathcal{S}_{2}}^{\prime}, l_{\mathcal{S}_{2} \mathcal{S}_{3}}\right)$. The other extremity of $e^{\prime}$ is $c^{\prime \prime}$ having label $\left(l_{\mathcal{O S}}, l_{\mathcal{O S}}\right.$, $\left.l_{\mathcal{O S}}, l_{\mathcal{S}_{1} \mathcal{S}_{2}}^{\prime}, l_{\mathcal{S}_{1} \mathcal{S}_{3}}^{\prime \prime}, l_{\mathcal{S}_{2} \mathcal{S}_{3}}\right)$ with $l_{\mathcal{S}_{1} \mathcal{S}_{3}} \neq l_{\mathcal{S}_{1} \mathcal{S}_{3}}^{\prime \prime}$.

In this manner, it is possible to turn around the 2-face of the connected component of $\mathcal{C}$ corresponding to the 4 -simple contact of label $\left(l_{\mathcal{O S}}, l_{\mathcal{O S}}, l_{\mathcal{O S}_{3}}\right.$, $l_{\mathcal{S}_{2} \mathcal{S}_{3}}$. The contacts $\mathcal{S}_{1}-\mathcal{S}_{2}$ and $\mathcal{S}_{1}-\mathcal{S}_{3}$ alternate on the edges of this 2-face. So, this 2-face must have at least four edges. 
The following properties are easily deduced:

- a 2 -face is at least a quadrilateral,

- a 3 -face is at least a parallelepiped,

- a 6 -face (i.e., a connected component) is at least a hyper-parallelepiped.

Moreover, Proposition 3 ensures that a 6 -simple contact is a convex vertex of $\mathcal{C}$. The following proposition summarizes these results about the shape and the size of such a connected component of $\mathcal{C}$ :

Proposition 4 In a system of three polygons in a polygonal environment, a connected component of $\mathcal{C}$ which does not contain double contact has at least 64 vertices and is convex.

Such a system exists, see Figure 3. In this example, there are exactly twelve labels of simple contact (two for each possibility of contacts, these simple contacts are marked by arrows on Figure 3 ), there are 64 ways to choose a 6 -simple contact label combining these twelve labels, each of these possibilities corresponds to a vertex of $\mathcal{C}$.

\subsection{Three Convex Polygons}

In some particular cases, the existence of a double contact can be proved.

Let $\mathcal{S}_{1}, \mathcal{S}_{2}$ and $\mathcal{S}_{3}$ be three convex polygons and $\mathcal{O}$ a polygonal environment. Let $c$, a vertex of $\mathcal{C}$ be a 6 -simple contact configuration. ¿From $c$, by breaking the contact $\mathcal{S}_{1}-\mathcal{S}_{2}$, it is possible to move along the corresponding edge. This movement in the configuration space corresponds to a relative translation moving $\mathcal{S}_{1}$ away from $\mathcal{S}_{2}$. Because $\mathcal{S}_{1}$ and $\mathcal{S}_{2}$ are both convex, it is impossible to restore the contact $\mathcal{S}_{1}-\mathcal{S}_{2}$ at the other extremity of the edge (even with another label), so it is necessary to introduce another contact already existing to restore a 6 -contact at the end of the edge. It follows a double contact configuration exists.

It is possible to prove a stronger result. From a 6 -contact $c$, one can move on the 3 -face corresponding to three contacts $\mathcal{S}_{i}-\mathcal{O}$ in the configuration space. Moving the configuration point along a half-line drawn on this 3 -face corresponds to a simultaneous translation of the three objects moving each convex polygon away from the two others. The intersection between the half-line and the 3 -face is a segment whose second extremity is at least a 4-contact (at most a 2 -face). As objects are moving away, the new contact is necessarily a contact between a convex polygon and the environment, and so a double contact. Unfortunately, it is not always possible to find another double contact, and Figure 4 shows an example where $\mathcal{S}_{1}$ is the only object able to get a double contact with $\mathcal{O}$. The different configurations drawn on Figure 4 represent the different vertices of $\mathcal{C}$. 


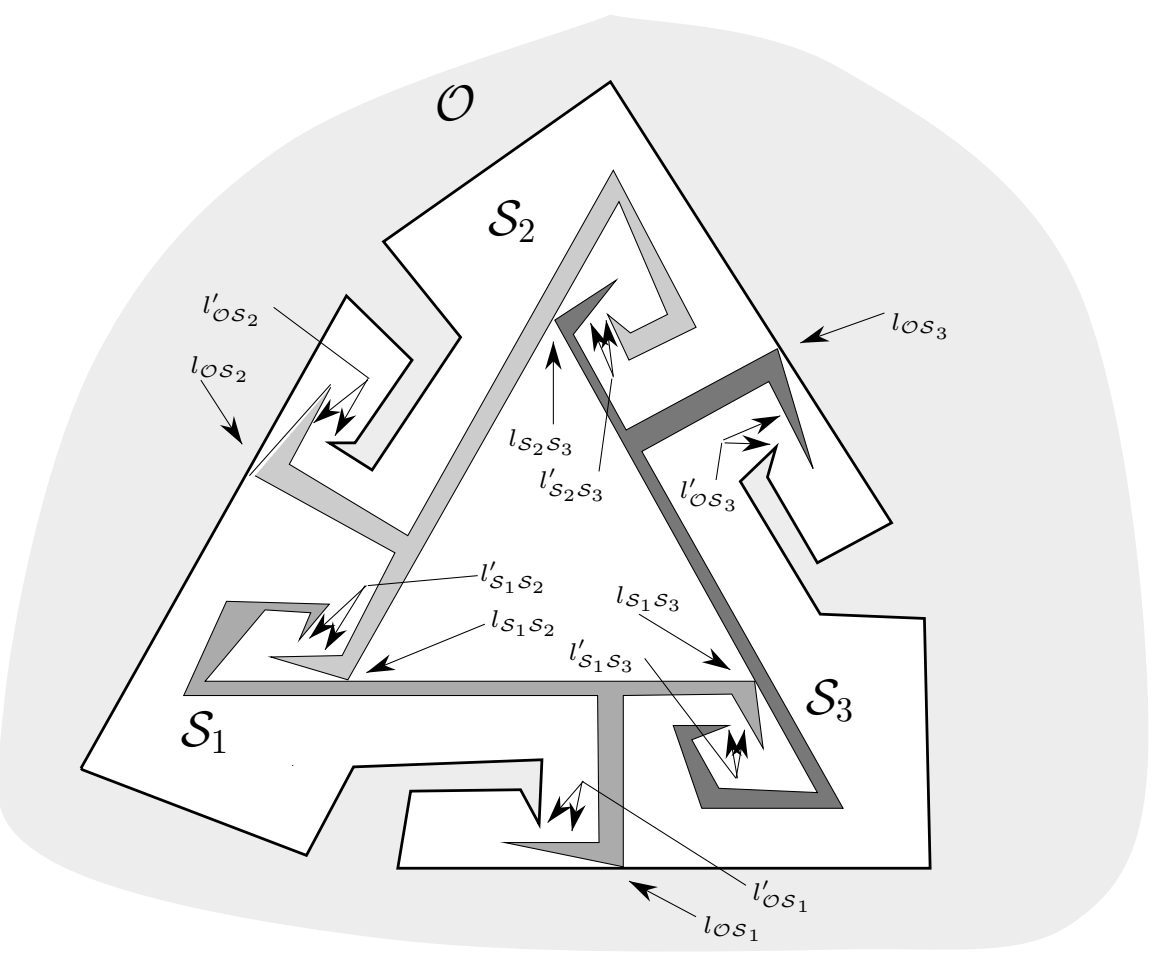

Figure 3: Free space without double contact

Proposition 5 In a system of three convex polygons in a polygonal environment, there exists in each connected component of $\mathcal{L}$, a configuration with a double contact between a polygon and the environment.

In the same way, it is possible to prove that:

Proposition 6 In a system of two convex polygons in a polygonal environment, there exists in each connected component of $\mathcal{L}$, a configuration where both polygons have a double contact with the environment.

\subsection{Three Polygons in a Rectangular Environment}

First, we prove an easy geometric lemma:

Lemma 7 Let $A, B$ and $C$ be three polygons in general position. If $r$ is a vertex of $A \cap(B \ominus C)$, then ( $r$ is a vertex of $A$ ) or $([b \in B, c \in C, r=b-c] \Rightarrow b$ is $a$ vertex of $B$ or $c$ is a vertex of $C$ ). 


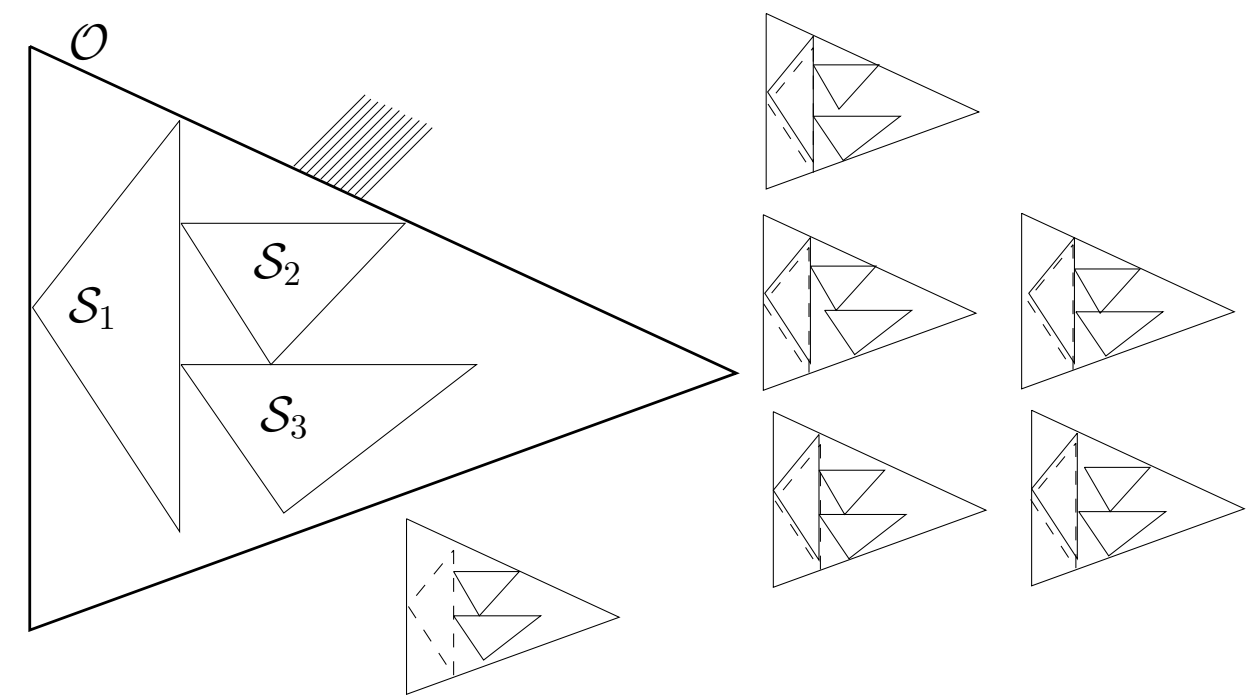

Figure 4: Three convex polygons with only one possibility of double contact

Proof: $r$ is either a vertex of $A$ or a vertex of $B \ominus C$ or a point of intersection of $A$ and an edge of $B \ominus C$. It is well known that an edge of $B \ominus C$ is the Minkowski difference of a vertex of $B$ and an edge of $C$ or an edge of $B$ and a vertex of $C$. This complete the proof.

Proposition 8 In a system of three polygons in a rectangular environment, there exists a configuration with a double contact (if $\mathcal{L} \neq \emptyset$ ).

Proof: If $\mathcal{C}$ is not empty, $\mathcal{R}_{12}$ is also not empty and has a vertex $c_{12}$. Using Lemma 7 and Equation (5), $c_{12}$ is a vertex of $\mathcal{U}_{12}$ or there exist $c_{13}$ and $c_{23}$ with $c_{12}=c_{13}-c_{23}$, and $c_{13}$ is a vertex of $\mathcal{U}_{13}$ or $c_{23}$ is a vertex of $\mathcal{U}_{23}$. So we have: there exists $c_{i j}$ vertex of $\mathcal{U}_{i j}$ corresponding to a free configuration in the complete system.

Applying Lemma 7 and Equation (3) again, we deduce that $c_{i j}$ is a vertex of $\mathcal{L}_{i j}$ (so there exists a double contact between $\mathcal{S}_{i}$ and $\mathcal{S}_{j}$ ), or $c_{i j}=c_{i}-c_{j}$ and $c_{k}\left(k=i\right.$ or $j$ ) is a vertex of $\mathcal{L}_{k}$ (so there exists a double contact between $\mathcal{S}_{k}$ and $\mathcal{O})$. 


\section{$\mathcal{O}$}

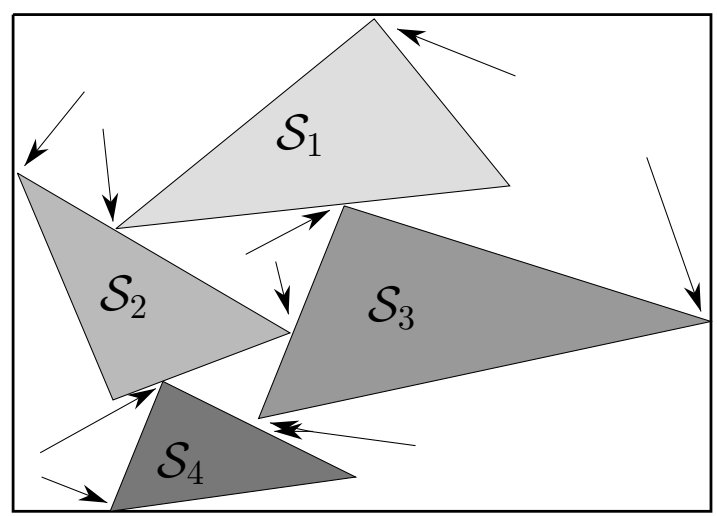

Figure 5: Four objects without double contact

\subsection{More than Three Objects}

As shown in Figure 5, it is possible to have a connected component of the free space without double contact, even for four convex polygons in a rectangular environment. In this example, the free space is a simplex, 9 labels of simple contact are marked on the figure, and the 9 vertices of $\mathcal{C}$ are defined by choosing 8 labels among these 9 .

\section{$5 \quad$ Placing Convex Polygons}

An algorithm to detect a free configuration (if there exists one) for two or three convex polygons in any polygonal environment can be deduced from the preceding section.

\subsection{Two Convex Polygons}

Proposition 6 assures that if $\mathcal{L}$ is not empty, there exists a 4 -contact configuration where both objects have a double contact with the environment. To detect such a configuration, we can use the following algorithm:

1. Compute $\mathcal{L}_{1}$ using Minkowski difference.

2. Compute $\mathcal{L}_{2}$.

3. Compute $\mathcal{L}_{12}$. 
4. For each pair of vertices $\left(c_{1}, c_{2}\right) \in \mathcal{L}_{1} \times \mathcal{L}_{2}$ test if $c_{1}-c_{2} \in \mathcal{L}_{12}$.

We can study the complexity of this algorithm. $n$ (resp. $m_{1}, m_{2}$ ) is the size of $\mathcal{O}\left(\right.$ resp. $\left.\mathcal{S}_{1}, \mathcal{S}_{2}\right)$.

- Step 1 consists of computing the Minkowski difference of a convex polygon and a general polygon. This can be done in time $O\left(n m_{1} \log n m_{1}\right)$ and the size of $\mathcal{L}_{1}$ is $O\left(n m_{1}\right)$.[7]

- In the same way Step 2 can be completed in time $O\left(n m_{2} \log n m_{2}\right)$ and the size of $\mathcal{L}_{2}$ is $O\left(n m_{2}\right)$.

- In Step 3, both polygons are convex. So, the Minkowski difference can be computed in time $O\left(m_{1}+m_{2}\right)$ and $\mathcal{L}_{12}$ is a convex polygon of size $O\left(m_{1}+m_{2}\right) \cdot[1]$

- Step 4 dominates the time complexity of the algorithm. The number of cases to be examined is $O\left(n^{2} m_{1} m_{2}\right)$. For each case we have to test whether a point is inside or outside a convex polygon of size $O\left(m_{1}+m_{2}\right)$ in time $O\left(\log \left(m_{1}+m_{2}\right)\right)=O\left(\log m_{1} m_{2}\right)$.

The time complexity of this algorithm is $O\left(n^{2} m_{1} m_{2} \log m_{1} m_{2}\right)$ which improves over the result of Avnaim and Boissonnat [2] $\left(O\left(n^{2} m_{1} m_{2} \log n m_{1} \log n m_{2}\right)\right)$.

\subsection{Three Convex Polygons}

From Proposition 5 we know that non-emptiness of $\mathcal{L}$ implies the existence of a 6 -contact configuration with a double contact between one object $\mathcal{S}_{i}$ and $\mathcal{O}$. The following algorithm searches for such a configuration:

1. Compute $\mathcal{L}_{1}, \mathcal{L}_{2}$ and $\mathcal{L}_{3}$.

2. For each vertex $c_{1}$ of $\mathcal{L}_{1}$, look for a free configuration of the system with two objects $\mathcal{S}_{2}$ and $\mathcal{S}_{3}$ in the environment $\mathcal{O} \cup \mathcal{S}_{1}^{c_{1}}$ using the algorithm of Section 5.1.

3. For each vertex $c_{2}$ of $\mathcal{L}_{2}$, look for a free configuration of the system with two objects $\mathcal{S}_{1}$ and $\mathcal{S}_{3}$ in the environment $\mathcal{O} \cup \mathcal{S}_{2}^{c_{2}}$ has a free configuration.

4. For each vertex $c_{3}$ of $\mathcal{L}_{3}$, look for a free configuration of the system with two objects $\mathcal{S}_{1}$ and $\mathcal{S}_{2}$ in the environment $\mathcal{O} \cup \mathcal{S}_{3}^{c_{3}}$.

The complexity of this algorithm is studied below.

- Step 1 can be computed in time $O\left(\sum_{i} n m_{i} \log n m_{i}\right)$ and the size of $\mathcal{L}_{i}$ is $O\left(n m_{i}\right) \cdot[7]$ 
- In Step 2, we have to examine $O\left(n m_{1}\right)$ cases. Each case is solved in time: $O\left(\left(n+m_{1}\right)^{2} m_{2} m_{3} \log m_{2} m_{3}\right)$ (see Section 5.1).

- Steps 3 and 4 are identical to the preceding one. The total time complexity of these two steps is

$O\left(n m_{2}\left(n+m_{2}\right)^{2} m_{1} m_{3} \log m_{1} m_{3}\right)$ and $O\left(n m_{3}\left(n+m_{3}\right)^{2} m_{1} m_{2} \log m_{1} m_{2}\right)$.

The complexity of the whole algorithm is:

$O\left(\sum_{i \neq j \neq k \neq i} n m_{i}\left(n+m_{i}\right)^{2} m_{j} m_{k} \log m_{j} m_{k}\right)$ which improves the preceding algorithm of Avnaim[3] whose complexity is

$O\left(n^{3} m_{1} m_{2} m_{3}\left(\left(m_{1}+m_{2}\right)\left(m_{1}+m_{3}\right) \log m_{1} m_{2} m_{3}+\log ^{2} n m_{1} m_{2} m_{3}\right)\right)$.

With the assumption that the sizes of the objects are $O(m)$ and that thay are smaller than the environment, we can summarize the total time complexity of this algorithm as $O\left(n^{3} m^{3} \log m\right)$ and the complexity of Avnaim's algorithm as $O\left(n^{3} m^{3} \log ^{2} n+n^{3} m^{5} \log m\right)$.

With the assumption that the objects have a constant size, our algorithms runs in $O\left(n^{3}\right)$ time, which is an improvement over the time complexity of $O\left(n^{3} \log ^{2} n\right)$ of Avnaim's algorithm[3] with the same assumption.

\section{Conclusion}

In this paper we have presented some results about the free space of several polygons translating in a polygonal environment. In the special cases of three convex polygonal objects translating in a polygonal environment, we have proved the existence of a double contact. We have also proved the existence of a double contact in the case of three polygonal objects translating in a rectangular environment. In the general case, we have shown an example of a non-empty free space with a convex connected component without double contact and presented some results about the minimum size of such a component. In the cases of two or three convex polygons, we have derived algorithms for computing a placement without collisions by computing double contacts. These algorithms are the fastest known for these problems. The case of more than three polygons does not present special properties concerning double contact configurations, as has been shown by an example.

The case of three polygons presents a special interest, because the number of degrees of freedom of the system is exactly the same than the number of possibilities of contacts (six). Generalizing to polygons translating and rotating in the plane or polyhedra translating in space, systems of five objects seems to play this special role since there are in this case three degrees of freedom per objects (and $15=3 \times 5=\left(\begin{array}{l}2 \\ 6\end{array}\right)$ ).

Another interesting problem in this area is the problem of establishing lower bounds for the problems considered in this paper. In some cases, a tight lower bound for the problem of computing all containment placements may be deduced 
from the size of the result in typical examples.[2, 4] However, for the problem of computing just one containment placement, or, to decide if the free space is empty or not, the question of computing tight lower bounds remains open.

\section{Acknowledgements}

The author would like to thank Francis Avnaim whose thesis initiated this work, Jean Ponce for a careful reading of the paper and Jean-Pierre Merlet for supplying his interactive drawing preparation system JPdraw . One of the anymous referees is gratefully acknowledged for having provided comments that helped improving the presentation and the clarity of the paper.

\section{References}

[1] L. J. Guibas, L. Ramshaw, and J. Stolfi. "A kinetic framework for computational geometry." In Proc. 24th Annu. IEEE Sympos. Found. Comput. Sci., pages 100-111, 1983.

[2] F. Avnaim and J.-D. Boissonnat. "Simultaneous containment of several polygons." In Proc. 3rd Annu. ACM Sympos. Comput. Geom., pages 242$250,1987$.

[3] F. Avnaim. "Placement et déplacement de formes rigides ou articulées." Thèse de doctorat en sciences, Université de Franche-Comté, Besançon, France, 1989.

[4] B. S. Baker, S. J. Fortune, and S. R. Mahaney. "Polygon containment under translation." J. Algorithms, 7:532-548, 1986.

[5] T. Lozano-Pérez and M. A. Wesley. "An algorithm for planning collisionfree paths among polyhedral obstacles." Commun. ACM, 22:560-570, 1979.

[6] H. Edelsbrunner. Algorithms in Combinatorial Geometry. Springer-Verlag, Heidelberg, West Germany, 1987.

[7] S. J. Fortune. "Fast algorithms for polygon containment." In Proc. 12th Internat. Colloq. Automat. Lang. Program., volume 194 of Lecture Notes in Computer Science, pages 189-198. Springer-Verlag, 1985.

[8] H. Edelsbrunner and E. P. Mücke. "Simulation of simplicity: a technique to cope with degenerate cases in geometric algorithms." ACM Trans. Graph., 9:66-104, 1990. 